\title{
What Powers the 2006 Outburst of the Symbiotic Star BF Cygni?
}

\author{
A. Skopal ${ }^{1}$, M. Sekeráś ${ }^{1}$, N. A. Tomov², M. T. Tomova ${ }^{2}$, T. N. Tarasova ${ }^{3}$, M. Wolf ${ }^{4}$ \\ ${ }^{1}$ Astronomical Institute of the Slovak Academy of Sciences, 05960 Tatranská Lomnica, Slovakia \\ ${ }^{2}$ Institute of Astronomy and NAO, Bulgarian Academy of Sciences, 4700 Smolyan, Bulgaria \\ ${ }^{3}$ Crimean Astrophysical Observatory, 298409 Nauchny, Crimea, Russia \\ ${ }^{4}$ Astronomical Institute, Charles University Prague, 18000 Praha 8, V Holešovičkách 2, Czech Republic \\ Corresponding author: skopal@ta3.sk
}

\begin{abstract}
BF Cygni is a classical symbiotic binary. Its optical light curve occasionally shows outbursts of the Z And-type, whose nature is not well understood. During the 2006 August, BF Cyg underwent the recent outburst, and continues its active phase to the present. The aim of this contribution is to determine the fundamental parameters of the hot component in the binary during the active phase. For this purpose we used a high- and low-resolution optical spectroscopy and the multicolour $U B V R_{\mathrm{C}} I_{\mathrm{C}}$ photometry. Our photometric monitoring revealed that a high level of the star's brightness lasts for unusually long time of $>7$ years. A sharp violet-shifted absorption component and broad emission wings in the $\mathrm{H} \alpha$ profile developed during the whole active phase. From 2009, our spectra revealed a bipolar ejection from the white dwarf (WD). Modelling the spectral energy distribution (SED) of the low-resolution spectra showed simultaneous presence of a warm $(<10000 \mathrm{~K})$ disk-like pseudophotosphere and a strong nebular component of radiation (emission measure of $\sim 10^{61} \mathrm{~cm}^{-3}$ ). The luminosity of the hot active object was estimated to $>5-8 \times 10^{3} L \odot$. Such high luminosity, sustained for the time of years, can be understood as a result of an enhanced transient accretion rate throughout a large disk, leading also to formation of collimated ejection from the WD.
\end{abstract}

Keywords: binaries: symbiotic - optical - spectroscopy - photometry - individual: BF Cyg.

\section{Introduction}

Symbiotic stars (SSs) are the largest interacting binary systems with known orbital periods in order of years. They consist of a cool giant and a WD accreting from the giant's wind. Accretion process heats up the WD to $1-2 \times 10^{5} \mathrm{~K}$ and makes it as luminous as a few times $10^{3} L_{\odot}$, whose photons ionize a large fraction of the neutral giant's wind, giving rise to nebular emission. As a result the spectrum of SSs consists of three basic components of radiation - two stellar and one nebular. If a symbiotic system releases its energy approximately at a constant rate and the temperature, it conforms the so-called quiescent phase. The stage, when the system brightens up in the optical by a few magnitudes and/or shows signatures of a mass-outflow, is named an active phase.

BF Cyg is an eclipsing symbiotic binary with an orbital period of $757.2 \mathrm{~d}$ (e.g. Pucinskas 1970; Fekel et al. 2001). The binary consists of a late-type M5 III giant (Mürset \& Schmid, 1999) and a hot luminous compact object (Mikolajewska et al., 1989). Its eclipsing nature was revealed by optical photometry of Skopal (1992). Historical light curve of BF Cyg is characterized by a slow, symbiotic-nova-type outburst (1895-1960), with superposed eruptions of the Z And-type (1920, 1989, 2006) and short-term flares (e.g. Skopal et al., 1997; Leibowitz \& Formiggini, 2006). The last, 1989 Z Andtype eruption was described by Cassatella et al. (1992), Skopal et al. (1997). The UV/optical continuum cooled to $\sim 20000 \mathrm{~K}$, and its source expanded to $\sim 7 R_{\odot}$ having a luminosity of $\sim 10000 L_{\odot}$. (see also Skopal, 2005). The line spectrum showed violet-shifted absorption lines indicating a mass outflow from the hot component at $100-500 \mathrm{~km} \mathrm{~s}^{-1}$.

The recent, 2006 outburst of BF Cyg was first reported by Munari et al. (2006). Spectroscopic observations by Sitko et al. (2006), Iijima (2006) and McKeever et al. (2011) indicated appearance of strong P-Cyg type of HI, HeI line profiles from the beginning of the outburst. Photometric observations indicated a high level of the star's brightness for much longer time than was observed for the previous, 1989-92, active phase (Siviero et al. 2012; Skopal et al., 2012). Recently, Skopal et al. (2013) reported an evidence of highly-collimated bipolar ejection from BF Cyg. 


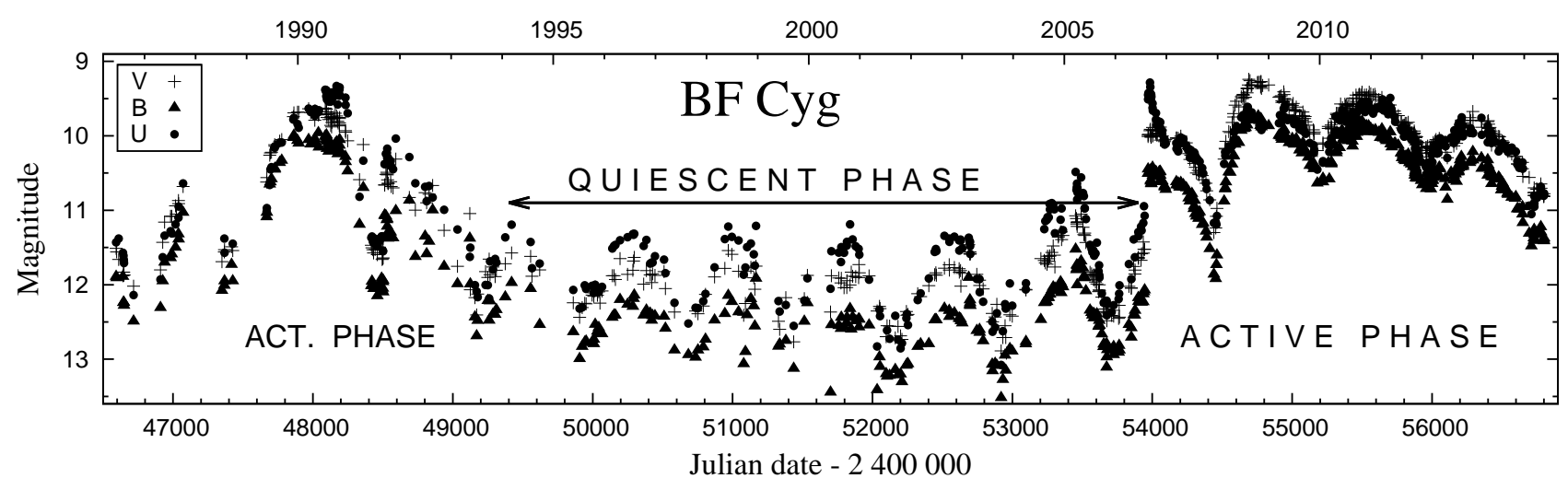

Figure 1: The $U B V$ light curves of BF Cyg from 1986 to the present. They cover the last, 1989-93, and the present, 2006-13, active phases. During the quiescent phase $(\sim 1994-2006)$, the star was by $2-3$ mag fainter, being characterized with the wave-like orbitally-related variation.

In this contribution we analyze our optical spectroscopy and $U B V R_{\mathrm{C}} I_{\mathrm{C}}$ photometry from the current active phase of $\mathrm{BF} \mathrm{Cyg}$, with the aim to determine fundamental parameters of the hot component. We point the problem of its high luminosity, which sustains for a long time of years.

\section{Observations}

Broad-band photoelectric $U B V$ and CCD $U B V R_{\mathrm{C}} I_{\mathrm{C}}$ photometry of BF Cyg was carried out by $0.6-\mathrm{m}$ telescopes at the Skalnaté Pleso and Stará Lesná observatories of Astronomical Institute of the Slovak Academy of Sciences (see Skopal et al. 2012 for details). The data are plotted in Fig. 1.

The high-resolution spectroscopy was carried out by the single dispersion slit spectrograph mounted at the coudé focus of the 2-m RCC telescope of the Rozhen National Astronomical Observatory and at the Ondřejov Observatory.

The low-resolution $(R \sim 1000)$ spectroscopic observations were secured by the $2.6-\mathrm{m}$ Shajn telescope, operated by the Crimean Astrophysical Observatory.

Spectroscopic observations were dereddened with $E_{\mathrm{B}-\mathrm{V}}=0.35$ and the resulting parameters were scaled to a distance of $3.8 \mathrm{kpc}$ (e.g. Skopal, 2005).

\section{Analysis and Results}

\subsection{Modelling the SED in the optical}

Assuming that the optical continuum consists of the three basic radiative components of radiation (see Sect. 1), the resulting flux in the continuum, $F(\lambda)$, can be expressed as their superposition,

$$
F(\lambda)=F_{\mathrm{WD}}(\lambda)+F_{\mathrm{N}}(\lambda)+F_{\mathrm{G}}(\lambda)
$$

where $F_{\mathrm{WD}}(\lambda)$ is the flux from the WD's pseudophotosphere, $F_{\mathrm{N}}(\lambda)$ is the flux from thermal plasma and $F_{\mathrm{G}}(\lambda)$ represents the flux from the giant. For effective temperatures, $T_{\mathrm{WD}}^{\mathrm{eff}} \sim 5000-10000 \mathrm{~K}$, an atmospheric model, $\mathcal{F}_{\lambda}\left(T_{\mathrm{WD}}^{\text {eff }}\right)$, is needed to fit the radiation of the warm pseudophotosphere. Otherwise, a simple blackbody radiation is satisfactory. The nebular radiation in the continuum can be approximated by processes of recombination and thermal bremsstrahlung in the hydrogen/helium plasma for Case B. Finally, radiation from the giant is represented by an appropriate synthetic spectrum, $\mathcal{F}_{\lambda}\left(T_{\mathrm{G}}^{\mathrm{eff}}\right)$. Then Eq. (1) can be expressed as,

$$
F(\lambda)=\theta_{\mathrm{WD}}^{2} \mathcal{F}_{\lambda}\left(T_{\mathrm{WD}}^{\mathrm{eff}}\right)+k_{\mathrm{N}} \varepsilon_{\lambda}\left(T_{\mathrm{e}}\right)+\theta_{\mathrm{G}}^{2} \mathcal{F}_{\lambda}\left(T_{\mathrm{G}}^{\mathrm{eff}}\right),
$$

where $\theta_{\mathrm{WD}}=R_{\mathrm{WD}} / d$ and $\theta_{\mathrm{G}}=R_{\mathrm{G}} / d$ are angular radii of the WD pseudophotosphere and the giant, respectively. The factor $k_{\mathrm{N}}$ (= the observed emission measure in $\mathrm{cm}^{-5}$ ) scales the volume emission coefficient $\varepsilon_{\lambda}\left(T_{\mathrm{e}}\right)$ of the nebular continuum to observations. Constant electron temperature, $T_{\mathrm{e}}$, throughout the nebula is assumed. Physical parameters of the model spectrum (2), $\theta_{\mathrm{WD}}, \theta_{\mathrm{G}}, T_{\mathrm{WD}}^{\mathrm{eff}}, T_{\mathrm{G}}^{\mathrm{eff}}, k_{\mathrm{N}}$ and $T_{\mathrm{e}}$, are given by the solution of Eq. (2), which corresponds to a minimum of the reduced $\chi^{2}$ function. The SED-fitting analysis was described by Skopal (2005) and Skopal et al., (2011).

\subsection{Physical parameters}

Example of a low-resolution $(3400-7000 \AA)$ spectrum, taken around a brightness maximum $(23 / 10 / 2008)$, is depicted in Fig. 2. The model SED shows that the spectrum is dominated by the radiation of the warm WD pseudophotosphere (denoted as the warm stellar component (WSC) by Skopal et al., 2011) and the nebular continuum. The light from the giant becomes more significant for $\lambda>6600 \AA$. 
The WSC is produced by a source with $T_{\mathrm{WD}}^{\mathrm{eff}} \sim 8500 \mathrm{~K}$, the effective radius of $\sim 25 R_{\odot}$ and the luminosity of $\sim 3000 L_{\odot}$. The nebular component was characterized with a high emission measure of $E M=4 \pi d^{2} \times k_{\mathrm{N}} \sim 2.6 \times 10^{61}(d / 3.8 \mathrm{kpc})^{2} \mathrm{~cm}^{-3}$, radiated at $T_{\mathrm{e}} \sim 30000 \mathrm{~K}$, which correspond to the luminosity $L_{\mathrm{N}} \sim 5100 L_{\odot}$. Thus the lower limit of the total hot component luminosity was $\sim 8100 L_{\odot}$, because only a fraction of the burning WD radiation can be converted to the WSC and the nebular emission.

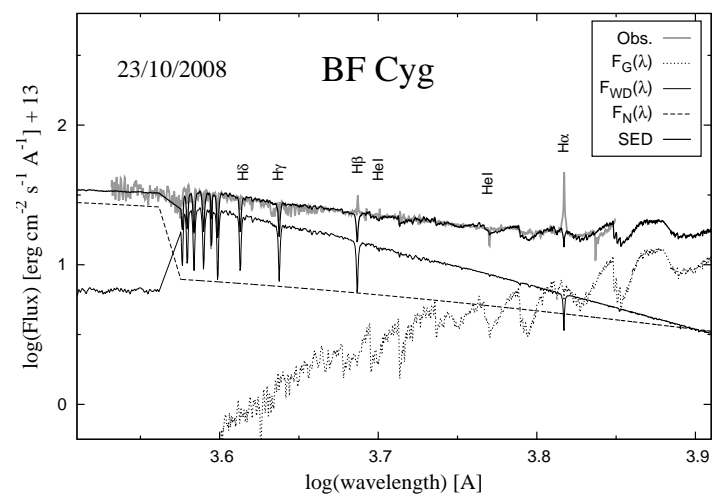

Figure 2: An example of the low-resolution spectrum (gray line) and its model (heavy solid line) taken during the 2006-13 active phase of BF Cyg, on 23/10/2008. The model SED and its components of radiation here represent a graphic form of Eq. (1) with the same denotation in keys.

\subsection{A disk-like shape of the WD pseudophotosphere}

The shape the WD pseudophotosphere cannot be spherical, because of the simultaneous presence of the strong nebular emission in the spectrum. If it were a sphere, its radiation would not be capable of giving rise to the observed nebular emission. On the other hand, the presence of the strong nebular emission in the spectrum constrains the presence of a hot ionizing source in the system. This type of the spectrum (called as twotemperature type) suggests that the WD pseudophotosphere has a form of a disk. When viewing the disk under a high inclination, its outer rim simulates the warm photosphere (producing the WSC), while the material above/below the disk is ionized by the hot central source and thus converts a fraction of its radiation to the nebular emission (see Skopal 2005 and Skopal et al. 2011 in detail).

\subsection{Collimated mass ejection}

Figure 3 shows evolution of the $\mathrm{H} \alpha$ profiles from the 2006 August eruption to 2013 April. The broad wings expanding to $\sim \pm 2000 \mathrm{~km} \mathrm{~s}^{-1}$ in $\mathrm{H} \alpha$ were present in all spectra. Significant variations were observed mainly at/around the line cores. (i) During the whole active phase, a sharp absorption developed on the blue side of the profile. (ii) During 2009, additional satellite emission components appeared at the position of a few times $\pm 100 \mathrm{~km} \mathrm{~s}^{-1}$ to the $\mathrm{H} \alpha$ emission core. (iii) During 2012 September, the satellite components were placed nearly symmetrically with respect to the $\mathrm{H} \alpha$ central emission.

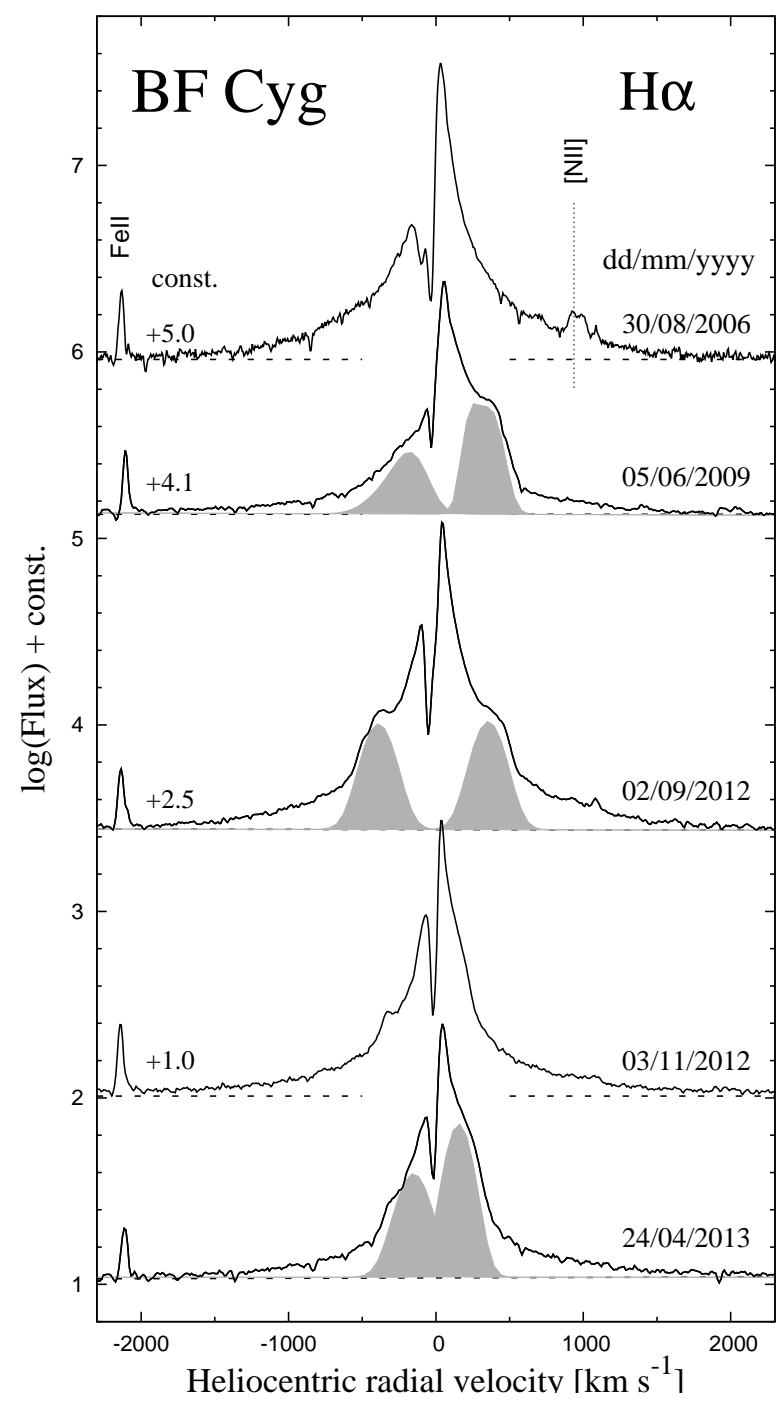

Figure 3: Evolution in the $\mathrm{H} \alpha$ line profile along the outburst. The filled curves represent the jet emission components (Sect. 3.4). Fluxes are in $10^{-13} \mathrm{erg} \mathrm{cm}^{-2} \mathrm{~s}^{-1} \AA^{-1}$.

Their radial velocities of $\pm \sim 370 \mathrm{~km} \mathrm{~s}^{-1}$ and fluxes of $\sim 1.4 \times 10^{-11} \mathrm{erg} \mathrm{cm}^{-2} \mathrm{~s}^{-1}$ were around a maximum (see Skopal et al., 2013 in detail). (iv) The presence of satellite components and their properties were unstable in the spectrum. During two months after their 
best pronounced stage (on 02/09/2012), they practically disappeared on 2012 November 3rd. However, in 2013 April, they re-appeared again (Fig. 3).

The relatively small width of the (well measured) satellite components $\left(F W H M \sim 245 \mathrm{~km} \mathrm{~s}^{-1}\right)$ and their radial velocities suggest that these emissions were produced by radiation of a highly collimated ejection by the central star.

\section{Concluding Remarks}

According to the elements of the spectoscopic orbit (Fekel et al., 2001), the mass of the WD in BF Cyg is as low as $\sim 0.55-0.6 M_{\odot}$. During the quiescent phase, the luminosity of the hot component was estimated to $\sim 10000 L_{\odot}$ for $d=3.8 \mathrm{kpc}$ (e.g. Mikolajewska et al., 1989). This quantity suggests that the source of such the energy output is caused by a stable hydrogen burning on the WD surface at the accretion rate of $\sim 1.4 \times 10^{-7} M_{\odot} \mathrm{yr}^{-1}$ for the $0.55-0.6 M_{\odot} \mathrm{WD}$ (e.g. Shen \& Bildsten, 2007). During active phase, the luminosity of the hot component can be $>10000 L_{\odot}$ (e.g. Cassatella et al., 1992), however, with difficulties of its precise determination as mentioned in Sect. 3.2. In addition, (i) a significant extension and thus cooling of the WD pseudophotosphere is indicated by modelling the SED (Sect. 3.2), (ii) an enhanced mass-loss rate from the WD is evidenced by the broad $\mathrm{H} \alpha$ wings with a violet-shifted absorption component, and (iii) an enhancement of the accretion rate onto the WD is required by the satellite emission components. The presence of bipolar jets confirms the presence of a disk around the accretor during the outburst. These observational properties are consistent with evolution of burning WDs in the H-R diagram, when the accretion rate increases above the stable burning regime. The accretion at $\approx 2 \times 10^{-7} M_{\odot} \mathrm{yr}^{-1}$ throughout the disk during the outburst can sustain the high luminosity of the burning WD at $\approx 10000 L_{\odot}$ (see Fig. 2 of Shen \& Bildsten, 2007).

The case of the current BF Cyg active phase lead us to a speculation that the simultaneous presence of the enhanced mass outflow and mass infall during some active phases of SSs can reflect a new type of the accretion process, which can sustain a high luminosity of their hot components for a long time of years. Similar properties with mass outflow/infall and jets were also observed during the 1977-1984 active phase of $\mathrm{CH}$ Cyg (e.g. Skopal et al. 2002). It is of interest to note that the enhanced mass outflow, sometimes followed with jet-like components, and emergence of a warm pseudophotosphere simulated by the irradiated disk are also observed during optical high states of supersoft X-ray sources (e.g. Southwell et al. 1996; Hutchings et al. 2002; Hachisu \& Kato, 2003).

\section{Acknowledgement}

This research was supported by the Slovak-Bulgarian Research and Development Cooperation project SKBG-0015-10, by the grant BSTC No. 01/14 BulgariaSlovakia, by the grant DO 02-85 of Bulgarian Scientific Research Fund, by the Research Program MSM0021620860 of the Ministry of Education of the Czech Republic and by a grant of the Slovak Academy of Sciences VEGA No. 2/0002/13.

\section{References}

[1] Cassatella A., et al.: 1992, A\&A 258, 368

[2] Fekel, F. C., et al.: 2001, AJ 121, 2219

[3] Hachisu, I., Kato, M.: 2003, ApJ, 588, 1003 doi:10.1086/374303

[4] Hutchings et al.: 2002, AJ, 124, 2833

[5] Iijima, T.: 2006, CBET No. 633

[6] Leibowitz, E. M., Formiggini, L.: 2006, MNRAS 366,675

doi:10.1111/j.1365-2966.2005.09895.x doi:10.1086/662076

[7] McKeever, J., et al.: 2011, PASP 123, 1062

[8] Mikolajewska, J., et al.: 1989, AJ 98, 1427

[9] Munari, U., et al.: 2006, CBET No. 596

[10] Mürset, U., Schmid, H. M.: 1999, A\&AS 137, 473 doi:10.1086/513457

[11] Pucinskas, A.: 1970, Bull. Vilnius Univ. Astron. Obs. No. 27,24

[12] Shen, K. J., Bildsten, L.: 2007, ApJ 660, 1444

[13] Sitko, M. L., et al.: 2006, IAUC No. 8746

[14] Siviero, A. et al.: 2012, Baltic Astron. 21, 188

[15] Skopal A.: 1992, IBVS No. 3780 doi:10.1093/mnras/292.3.703

[16] Skopal, A.: 2005, A\&A 440, 995 doi:10.1046/j.1365-8711.2002.05715.x

[17] Skopal, A., et al.: 1997, MNRAS 292, 703

[18] Skopal, A., et al.: 2002, MNRAS 335, 1109 doi:10.1002/asna. 201111655

[19] Skopal, A., et al.: 2011, A\&A 536, id. A27

[20] Skopal, A., et al.: 2012, Astron. Nachr. 333, 242 doi:10.1086/177931 
[21] Skopal, A., et al.: 2013, A\&A 551, id. L10

[22] Southwell et al.: 1996, ApJ, 470, 1065

\section{DISCUSSION}

DMITRY BISIKALO: If you have a wind from the WD during the quiescence, how can you accumulate matter to form a huge accretion disk?
AUGUSTIN SKOPAL: The presence of a wind from the WD is indicated even during quiescent phase by, for example, the broad $\mathrm{H}_{\alpha}$ wings. The presence of a large disk-like formation during active phases is observationally confirmed. However, its creation is not well understood yet. 